\title{
Conhecimento de profissionais da saúde em hipertensão arterial sistêmica: intervenção com cartilha ilustrativa
}

\author{
Knowledge of health professionals in systemic arterial hypertension: intervention with an \\ illustrative booklet
}

\author{
Conocimiento de los profesionales de la salud en hipertensión arterial sistémica: \\ intervención con folleto ilustrativo
}

Nathália Bianca Gomes Nóbrega ${ }^{1 *}$, Andreza Josiany Aires de Farias², José Rocha Gouveia Neto², Mayza Luzia dos Santos Neves ${ }^{3}$, Amanda Alana de Miranda Alves ${ }^{2}$, Ana Letícia Alves de Carvalho², Érica Pereira Alves ${ }^{2}$, Daniele Mamédio de Andrade ${ }^{2}$, Letícia Lany de Miranda Medeiros ${ }^{2}$, Taciana da Costa Farias Almeida4.

\section{RESUMO}

Objetivo: Avaliar o efeito de uma capacitação com cartilha ilustrativa no conhecimento dos profissionais sobre a hipertensão arterial e o atendimento ao paciente hipertenso. Métodos: Pesquisa quantitativa descritiva do tipo pesquisa-ação, com observação dos efeitos produzidos por ações de intervenção. Este estudo foi dividido em dois momentos, o primeiro destinado à resolução de questionário acerca da Hipertensão Arterial Sistêmica, do qual participaram 27 profissionais e o segundo momento, uma capacitação na modalidade de roda de conversa junto ao uso de cartilha ilustrativa. Em seguida, aplicou-se novamente o questionário, colaborando com esta etapa 20 participantes. O conhecimento, portanto, foi avaliado com base em respostas de questionário aplicado antes e após intervenções educativas. Resultados: Houve maior frequência de acertos na resolução do questionário após ações de intervenção, com melhora do conhecimento no tocante à hipertensão e ao cuidado do paciente hipertenso. A capacitação foi referida pela totalidade $20(100 \%)$ dos participantes que responderam a segunda aplicação do questionário como favorável ao aprendizado. Conclusão: a educação permanente se mostrou satisfatória por promover melhora do nível de conhecimento no que se trata a Hipertensão Arterial, assim como as estratégias utilizadas viáveis para qualificação profissional.

Palavras-chave: Hipertensão, Atenção primária à saúde, Educação continuada.

\begin{abstract}
Objective: To evaluate the effect of training with an illustrative booklet on the knowledge of professionals on the care of hypertensive patients. Methods: Quantitative descriptive research of the type of action research, with observation of the effects produced by intervention actions. This study was divided into two moments, the first aimed at solving a questionnaire about Systemic Arterial Hypertension, in which 27 professionals participated, and the second moment a training in the modality of conversation with the use of an illustrative booklet and domino game, in Then, the questionnaire was applied again, collaborating with these stage 20 participants. Knowledge, therefore, was assessed based on responses to a questionnaire applied before and after educational interventions. Results: There was a higher frequency of correct answers in solving the questionnaire after intervention actions. The training was mentioned by all $20(100 \%)$ of the subjects who answered the second questionnaire application as favorable to learning. Conclusion: Permanent education proved to be satisfactory for promoting an improvement in the level of knowledge regarding Arterial Hypertension, as well as the viable strategies used for professional qualification.
\end{abstract}

Keywords: Hypertension, Primary health care, Education continuing.

\footnotetext{
${ }^{1}$ Centro Universitário Tabosa de Almeida (ASCES- UNITA), Caruaru - PE.

*E-mail: natnobrega@hotmail.com

2 Universidade Federal de Campina Grande (UFCG), Campina Grande - PB.

3 Universidade Estadual de Campinas (Unicamp), Campinas - SP.

${ }^{4}$ Universidade Federal da Paraíba (UFPB), João Pessoa - PB.
} 


\section{RESUMEN}

Objetivo: Evaluar el efecto de la formación con un folleto ilustrativo sobre el conocimiento de los profesionales en la atención de pacientes hipertensos. Métodos: Investigación descriptiva cuantitativa del tipo de investigación acción, con observación de los efectos producidos por las acciones de intervención. Este estudio se dividió en dos momentos, el primero dirigido a la resolución de un cuestionario sobre Hipertensión Arterial Sistémica, en el que participaron 27 profesionales, y el segundo momento un entrenamiento en la modalidad de conversación con el uso de un folleto ilustrativo, Luego, se aplicó nuevamente el cuestionario, colaborando con esta etapa 20 participantes. El conocimiento, por lo tanto, se evaluó con base en las respuestas a un cuestionario aplicado antes y después de las intervenciones educativas. Resultados: Hubo mayor frecuencia de aciertos en la resolución del cuestionario luego de las acciones de intervención. La capacitación fue mencionada por los $20(100 \%)$ de los sujetos que respondieron la segunda solicitud del cuestionario como favorable al aprendizaje. Conclusión: La educación permanente resultó ser satisfactoria para promover una mejora en el nivel de conocimientos sobre Hipertensión Arterial, así como las estrategias viables utilizadas para la calificación profesional.

Palabras clave: Hipertensión, Atención primaria de salud, Educación continua.

\section{INTRODUÇÃO}

A Hipertensão Arterial Sistêmica (HAS) é uma condição clínica que apresenta correlação direta e progressiva sobre o desenvolvimento das doenças cardiovasculares, principal causa de morte no Brasil. Possui alta prevalência e morbimortalidade, baixas taxas de controle e custos socioeconômicos significativos, sendo considerada um grave problema de saúde pública. O diagnóstico precoce, tratamento contínuo, controle dos níveis pressóricos e a diminuição dos fatores de risco através da modificação do estilo de vida e/ou uso regular de medicamentos estão entre as medidas de controle para impedir a evolução da doença (MALACHIAS MVB, et al., 2016).

Nessa perspectiva, a Atenção Primária à Saúde (APS) surge como porta de entrada do Sistema Único de Saúde (SUS) e coordenadora do cuidado a esta clientela, uma vez que propicia, além da promoção e prevenção do desenvolvimento da doença e de suas complicações, a recuperação da saúde ao oferecer atendimento e acompanhamento de usuários diagnosticados (MELO JD, et al., 2016).

Estas ações são executadas prioritariamente pela Estratégia Saúde da Família (ESF), representada pelas Unidades Básicas de Saúde da Família (UBSF), por seu caráter potencial de aprofundar os princípios, diretrizes e fundamentos da APS sob a forma de trabalho em equipe, formação de vínculo e responsabilização da população adscrita (CAMARGO RAAD, et al., 2013).

Para tanto, atingir resultados positivos e de impacto requer das equipes envolvidas no cuidado às doenças crônicas o discernimento sobre os diversos determinantes socioeconômicos e culturais, organizando as ações com base nas reais necessidades de saúde do indivíduo, família e comunidade (BRASIL, 2014). Os profissionais envolvidos no atendimento ao usuário devem possuir preparo técnico-científico para oferecer uma assistência qualificada. Sob essa perspectiva, surge a proposição de Educação Permanente em Saúde, que remete ao ensino para profissionais em seus espaços de trabalho, com vista a aquisição de conhecimentos, habilidades e atitudes para melhoria da prática profissional (FALKENBERG MB, et al., 2014).

Instituída pelo Ministério da Saúde em 13 de fevereiro de 2004, a Portaria no 198/GM estabelece a Política Nacional de Educação Permanente em Saúde, uma estratégia do SUS para a formação e o desenvolvimento de trabalhadores para o setor através da articulação entre ensino, serviço e comunidade. Tal política pautase em demandas e necessidades apresentadas pela população e dificuldades do processo de trabalho para, dessa forma, desenvolver ações mais integrais e efetivas, que garantam maior resolubilidade aos serviços. Assim, além de superar deficiências do sistema, corrobora para o fortalecimento da participação e controle social (BRASIL, 2004).

Portanto, essa metodologia possibilita aliar a formação profissional com a realidade dos serviços, promovendo a construção de novas práticas em benefício da saúde da população (BRASIL, 2014). Uma revisão de literatura acerca da atuação do enfermeiro à pessoa hipertensa na ESF revelou que, na prática 
assistencial, o cumprimento de recomendações estabelecidas por diretrizes e protocolos ainda é, por grande parte dos profissionais, inconsistente e empírico, o que presume um cuidado limitado (MELO LM, et al., 2015).

Em estudo de Pessoa MS, et al. (2018), realizado em uma das unidades a ser estudada, identificou-se um alto número de hipertensos sendo acompanhados na UBSF. Participaram do estudo 112 hipertensos, e ao aplicar a escala de adesão terapêutica de Morisky e Green de oito itens, apenas $08(4,5 \%)$ hipertensos apresentavam alta adesão ao tratamento, e $02(1,8 \%)$ apresentavam ótima qualidade de vida, de acordo com a escala Minichal-Brasil que avalia Qualidade de Vida.

Diante dos dados da pesquisa supracitada que apontou na UBSF alta prevalência de HAS, baixa aderência terapêutica e qualidade de vida dos usuários, infere-se que o seguimento terapêutico insatisfatório pode estar associado ao baixo conhecimento dos profissionais, assim como déficit no acolhimento a clientela hipertensa por parte dos profissionais envolvidos com o cuidado. Portanto, uma gestão de cuidado sistemática é favorável a maior adesão ao tratamento, a uma melhor qualidade de vida e, consequentemente, a redução da morbimortalidade e de gastos públicos.

Ademais, estes tipos de levantamentos são relevantes no intuito de buscar cada vez mais por melhorias na assistência à saúde da pessoa que apresenta o diagnóstico de hipertensão arterial sistêmica na APS. Diante das considerações, se torna viável e necessária a capacitação desses profissionais, a qual partirá do levantamento do conhecimento destes sobre o atendimento a essa clientela. Assim, este estudo apresenta o objetivo de avaliar o efeito de uma capacitação com cartilha ilustrativa no conhecimento dos profissionais sobre a hipertensão arterial e o atendimento ao paciente hipertenso.

\section{MÉTODOS}

Este estudo faz parte de um projeto maior intitulado "Adesão terapêutica e qualidade de vida de indivíduos hipertensos assistidos em uma Unidade de Saúde da Família". Trata-se de uma pesquisa quantitativa descritiva do tipo pesquisa-ação, com observação dos efeitos produzidos por uma ação de intervenção.

A pesquisa-ação é um dos inúmeros tipos de investigação-ação, cujo processo pode ser descrito em um ciclo, no qual planeja-se, implementa-se, descreve-se e avalia-se a mudança para melhoria no campo da prática. Frequentemente se produzem dados sobre os efeitos de uma mudança da prática antes e após implementação. Ademais, essa metodologia se caracteriza como inovadora, participativa, problematizadora intervencionista e proativa estrategicamente (Figura 1) (TRIPP D, 2005).

Figura 1 - Representação em quatro fases do ciclo básico da investigação-ação.

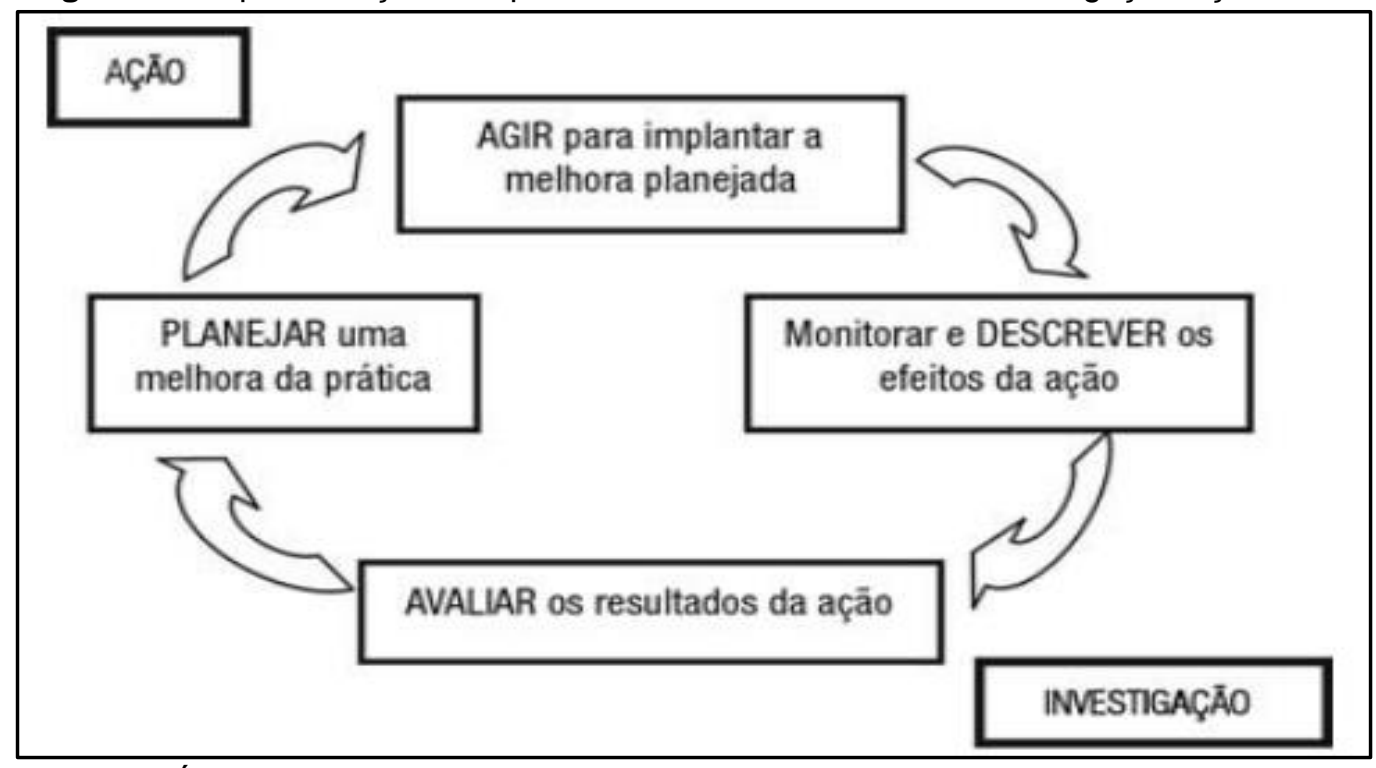

Fonte: NÓBREGA NBG, et al., 2020; extraído de TRIPP D, 2005. 
O estudo foi desenvolvido em duas Unidades Básicas de Saúde da Família situadas em município de Paraíba. A população do presente estudo foi composta pelos profissionais que atuam nas respectivas UBSF, que corresponde a quatro enfermeiras, três médicos, três técnicos de enfermagem, um odontólogo, uma auxiliar de saúde bucal, uma auxiliar de farmácia, uma assistente social, três recepcionistas, duas auxiliares de serviços gerais, dois vigilantes e 14 agentes comunitários de saúde, totalizando 35 profissionais.

A seleção da amostra deu-se por conveniência, considerando os profissionais mais prontamente acessíveis ao estudo, de acordo com a disponibilidade da pesquisadora. Para alcançar o maior número de participantes, a pesquisadora optou por comparecer ao local de pesquisa o maior número de vezes possível, no intuito de abordar a população. No entanto, obteve-se como amostra, 27 e 20 sujeitos, no primeiro e segundo momento, respectivamente.

Como critério de inclusão utilizou-se: ser profissional atuante na UBSF, estar presente e possuir disposição para responder o questionário, participar das ações de intervenção e entrar em contato diariamente com usuários com hipertensão arterial. A coleta de dados ocorreu nos momentos de pré e pós intervenção, nos locais de trabalho (duas UBSF), no período de setembro a novembro de 2017.

\section{Primeiro momento}

Inicialmente, os participantes foram orientados sobre os objetivos da pesquisa e convidados a participar do estudo e, em caso de aceite, eles recebiam o Termo de Consentimento Livre e Esclarecido (TCLE). Após aceite e assinatura do TCLE, eles foram orientados a não consultarem os demais colegas e não comunicarem as respostas para que a avaliação do conhecimento fosse específica e individual.

Utilizou-se de um questionário com 23 itens, destes, seis questões estavam relacionadas ao atendimento ao usuário com diagnóstico de HAS, sendo três discursivas e auto avaliativas, 13 questões de múltipla escolha relativas ao conhecimento sobre a hipertensão e uma sobre a necessidade de capacitação. Este instrumento foi elaborado pelos pesquisadores, conforme o Caderno de Atenção Básica ํo 37 - Estratégias para o cuidado da pessoa com doença crônica - Hipertensão Arterial Sistêmica. Para análise de seu conteúdo e adequação a população alvo da pesquisa, foi aplicado em pré-teste a população semelhante, composta por cinco profissionais (dois enfermeiros, um técnico de enfermagem, um médico e um agente comunitário de saúde) que atuavam em uma UBSF semelhantes às estudadas.

\section{Segundo momento}

No segundo momento, realizou-se educação permanente com base no Caderno de Atenção Básica oㅡ 37, Estratégias para o cuidado da pessoa com doença crônica - Hipertensão, e na VII Diretriz Brasileira de Hipertensão, priorizando os erros cometidos na resolução do questionário no primeiro momento. Optou-se por formas ativas de aprendizado coletivo, utilizando-se como metodologia a roda de conversa junto a cartilha ilustrativa elaborada. Nesse mesmo dia, houve nova aplicação do questionário, para avaliar o impacto das ações sob a forma de aquisição do conhecimento, acrescido de questões acerca das intervenções a fim de obter a avalição da estratégia utilizada.

Os dados dos questionários foram digitados em planilha Microsoft Excel e posteriormente inseridos no Programa SPSS versão 21.0 para análise descritiva e inferencial, utilizando os testes estatísticos de acordo com as variáveis investigadas. Conforme recomendações da resolução 466-2012 do Conselho Nacional de Saúde, que regulamenta pesquisas envolvendo seres humanos, o projeto de pesquisa foi submetido ao Comitê de Ética em Pesquisa (CEP), recebendo aprovação (Parecer número 1.313.566 e CAAE: 38249314.0.0000.5182).

\section{RESULTADOS}

\section{Primeiro momento}

Participaram do estudo 27 profissionais de duas Unidades Básicas de Saúde da Família. Em razão de inacessibilidade por férias, folgas e licenças ou negativa de participação na pesquisa de oito profissionais, não foi possível a participação da população. 
Houve predomínio do sexo feminino $(70,4 \%)$ e média de 40,59 anos. Dentre os participantes, $(40,7 \%)$ eram agentes comunitários de saúde, $(11,1 \%)$ enfermeiros, $(11,1 \%)$ recepcionistas, $(7,4 \%)$ técnicos de enfermagem, $(7,4 \%)$ vigilantes, $(3,7 \%)$ assistente social, $(3,7 \%)$ médico, $(3,7 \%)$ odontólogo, $(3,7 \%)$ auxiliar de farmácia, (3,7\%) auxiliar de saúde bucal, e (3,7\%) auxiliar de serviços gerais.

Constatou-se que a totalidade (100\%) possuía contato ou atendia hipertensos em seu local de trabalho, porém apenas $(66,7 \%)$ afirmou reconhecer os hipertensos que buscavam atendimento em sua unidade, e $(33,3 \%)$ referiram conhecer somente alguns deles.

A realização de programas específicos para este público foi referida por $(51,9 \%)$ profissionais, e foram descritos como HiperDia (29,6\%), marcação de consulta (3,7\%), atendimento $(3,7 \%)$, orientação $(3,7 \%)$, acompanhamento $(3,7 \%)$, com as seguintes frequências relatadas: mensalmente $(25,9 \%)$, semanalmente $(11,1 \%)$ e diariamente $(11,1 \%)$ vezes. Ao serem arguidos sobre o atendimento oferecido a população hipertensa, observaram-se as respostas descritas abaixo (Figura 2).

Figura 2 - Auto - avaliação do atendimento oferecido ao hipertenso.

\section{EÓTIMO $=$ BOM $=$ REGULAR $=$ RUIM}

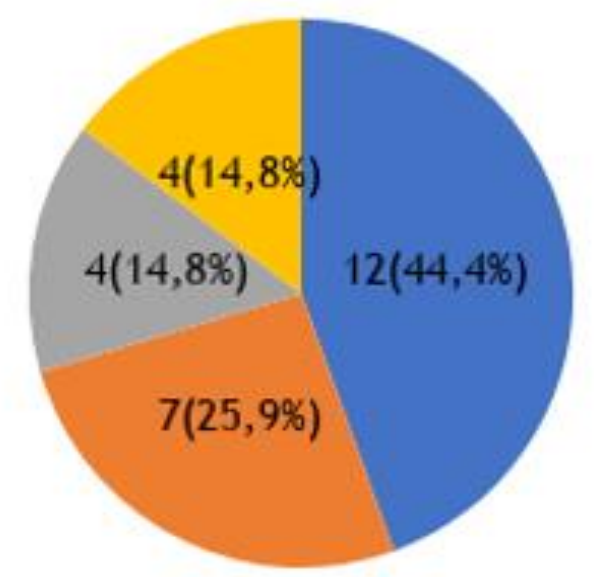

Fonte: NÓBREGA NBG, et al., 2020.

As justificativas para esta autoavaliação variaram, no aspecto positivo, em atendimento satisfatório $(29,6 \%)$, resolutividade $(7,4 \%)$ e humanização $(3,7 \%)$, e negativamente, em programa saúde em casa $(18,5 \%)$, falta de insumos e recursos $(11,1 \%)$, grande demanda $(11,1 \%)$, localização inadequada da UBSF $(7,4 \%)$, médico particular $(3,7 \%)$, falta de contato $(3,7 \%)$, e $(11,1 \%)$ não responderam à questão. Em relação as atividades profissionais direcionadas aos hipertensos, foram citadas respostas semelhantes, destacandose as descritas na (Tabela 1).

Tabela 1 - Distribuição das atividades profissionais citadas no atendimento ao hipertenso. $n=27$.

\begin{tabular}{cc}
\hline Atividades desenvolvidas & $\mathbf{n}(\%)$ \\
\hline Solicitação de exames & $5(18,5 \%)$ \\
Consulta médica e de enfermagem mensal & $4(14,8 \%)$ \\
Oferecer orientações & $3(11,1 \%)$ \\
Acolher & $2(7,4 \%)$ \\
Acompanhamento e atividades semanais & $2(7,4 \%)$ \\
Realizar encaminhamentos & $2(7,4 \%)$ \\
Verificação da Pressão Arterial & $2(7,4 \%)$ \\
Consulta odontológica mensal & $1(3,7 \%)$ \\
Convidar para atividades & $1(3,7 \%)$ \\
Ações de educação em saúde & $1(3,7 \%)$ \\
Entregar medicação & $1(3,7 \%)$ \\
\hline
\end{tabular}

Fonte: Nóbrega NBG, et al., 2020. 
Preocupados com satisfação dos profissionais em relação ao seu atendimento à clientela hipertensa, optou-se por aplicar uma escala de satisfação acerca deste atendimento, cujos resultados estão apresentados (Figura 3).

Figura 3 - Representatividade do grau de satisfação dos profissionais quanto ao atendimento ofertado. $\mathrm{n}=27$.

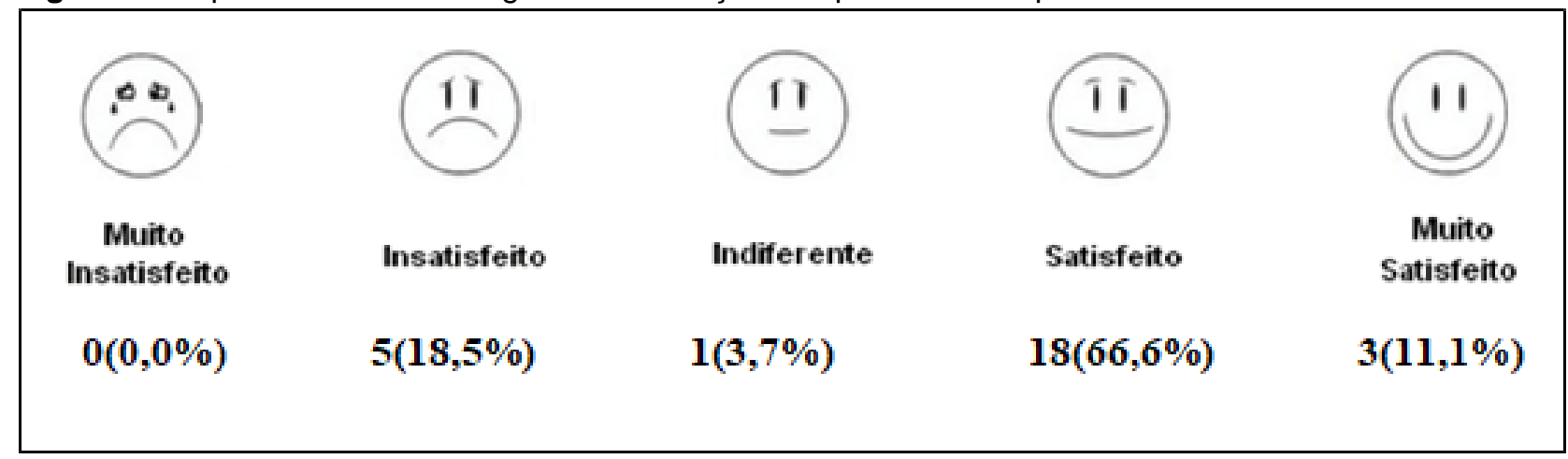

Fonte: NÓBREGA NBG, et al., 2020.

Dentre os aspectos que poderiam melhorar a satisfação disposta na Figura 4, foram mencionados: regularidade na entrega e acesso às medicações $11(40,7 \%)$, melhoria na marcação de exames e encaminhamentos $(22,2 \%)$, adesão ao tratamento $(14,8 \%)$, cogestão $(7,4 \%)$, conhecimento $(7,4 \%)$, acompanhamento contínuo $(7,4 \%)$ e acolhimento (3,7\%).

Enquanto $(11,1 \%)$ referiram não necessitar de melhora. Salienta-se que um participante não respondeu a esta pergunta. Para melhoria do atendimento ao hipertenso, 16(59,3\%) afirmou que necessita de capacitação, dos quais $(18,5 \%)$ sugeriu a palestra, $(3,7 \%)$ considerou como sugestão de estratégia a metodologia ativa e $(3,7 \%)$ técnicas persuasivas para com o paciente.

\section{Segundo momento}

Neste dia esteve presente a pesquisadora e três integrantes do Grupo de Estudos em Medida da Pressão Arterial (GEMPA) para a condução da educação permanente. Considerando as estratégias de capacitação sugeridas pelos profissionais em resposta ao questionário, desenrolou-se uma roda de conversa com simulação da realidade e, através dessa metodologia ativa, todos puderam interagir, fazer comentários, trocar experiências e tirar suas dúvidas.

Foram introduzidos conceitos, fatores de risco, complicações, classificação, diagnóstico e tratamento da hipertensão, ao mesmo tempo em que passava por eles uma cartilha ilustrativa referente à temática. A medida da pressão arterial também foi abordada por meio de simulação realística.

Em uma situação hipotética, um paciente chegava à unidade para verificar a PA e então pedia-se que um voluntário demonstrasse o preparo do paciente e a aferição adequada. A partir daí, indagava-se sobre o que estaria certo ou não no procedimento.

Foi um momento em que eles puderam refletir, discutir suas vivências e praticar a medida uns nos outros. Por fim, aplicou-se novamente o questionário. Ao aplicar as 14 questões acerca da hipertensão e cuidados ao hipertenso, observou-se as seguintes assertivas antes e após a intervenção, dispostas (Tabela 2).

Nesta segunda aplicação, foi questionado aos profissionais se a Educação Permanente contribuiu para aprimorar o conhecimento, e o total de (100\%) foi concordante. 
Tabela 2 - Frequência de acertos apresentadas na resolução do questionário nos momentos de pré e pós capacitação. $n=27$ (pré-intervenção) e $n=20$ (pós-intervenção).

\begin{tabular}{ccc}
\hline Questão & $\begin{array}{c}\text { Acertos } \mathbf{n}(\%) \\
\text { (Pré capacitação) }\end{array}$ & $\begin{array}{c}\text { Acertos n (\%) } \\
\text { (Pós capacitação) }{ }^{\#}\end{array}$ \\
\hline Definição de HAS & $20(74,1)$ & $19(95)$ \\
Prevalência & $09(33,3)$ & $08(40)$ \\
Idade que mais ocorre & $18(66,7)$ & $15(75)$ \\
Complicações & $26(96,3)$ & $19(95)$ \\
Fatores de Risco & $26(96,3)$ & $20(100)$ \\
Primeira verificação da PA & $12(44,4)$ & $15(75)$ \\
Classificação da PA & $11(40,7)$ & $20(100)$ \\
Valor que caracteriza a hipertensão & $17(63,0)$ & $13(65)$ \\
Fechamento do diagnóstico de HAS & $14(51,9)$ & $17(85)$ \\
Medidas de controle da HAS & $27(100,0)$ & $20(100)$ \\
Dados a serem avaliados & $27(100,0)$ & $20(100)$ \\
O que deve ser verificado na consulta & $19(70,4)$ & $17(85)$ \\
Período de coleta de exames de rotina & $15(55,6)$ & $19(95)$ \\
Equipe envolvida no atendimento & $26(96,3)$ & $20(100)$ \\
\hline
\end{tabular}

Legenda: *Responderam ao instrumento pré-intervenção $(n=27)$ / "Responderam ao instrumento pósintervenção $(n=20)$.

Fonte: NÓBREGA NBG, et al., 2020.

\section{DISCUSSÃO}

Verificou-se que em relação ao conhecimento de profissionais da APS sobre o atendimento ao hipertenso, todos que responderam ao questionário afirmaram ter algum tipo de contato com pacientes hipertensos. Isto confere que todos os profissionais criam relações, em maior ou menor grau de proximidade, com aqueles que buscam atendimento nas UBSF. Reforça ainda, as recomendações do Ministério da Saúde quanto às ações de caráter multiprofissional no combate a HAS (BRASIL, 2013).

Este dado confronta, porém, com a baixa porcentagem daqueles que reconhecem os hipertensos em sua unidade, o que pode estar relacionado ao crescente número de pacientes hipertensos, tornando os profissionais responsáveis por uma demanda cada vez maior.

A avaliação do atendimento oferecido na unidade, de forma geral, foi avaliada como positiva, pois esse foi considerado humanizado e resolutivo, pontos fundamentais que garantem boas práticas de saúde, satisfação profissional e da clientela. Para aqueles que fizeram avaliação negativa, a justificativa para o atendimento regular ou ruim foi, sobretudo, o Programa Saúde em Casa. O programa possibilita que hipertensos recebam mensalmente seus medicamentos nas suas residências, sendo que, a cada 90 dias o paciente precisa passar por avaliação médica na unidade e a cada 180 dias a prescrição precisa ser renovada (CODEHCOM, 2017).

Essa iniciativa propõe oferecer maior comodidade aos usuários, entretanto, questiona-se até que ponto o benefício do conforto sobrepõe a avalição contínua dos níveis pressóricos e da adesão terapêutica. Além disso, o comparecimento eventual à UBSF por parte dos usuários pode provocar distanciamento e perda de vínculo com a equipe.

Quanto à insatisfação pessoal relacionada ao atendimento prestado, recaiu sobre aspectos de gestão, como a dificuldade na marcação de exames e consultas, irregularidade na entrega de medicações e sobre a falta de adesão ao tratamento por parte dos usuários. Embora alguns aspectos gerenciais e organizacionais citados nesse estudo não dependam exclusivamente dos profissionais, devem ser refletidos para impulsionar melhorias, pois a má estrutura e organização dos serviços de saúde podem criar obstáculos tanto aos 
trabalhadores, que são impedidos de exercerem suas funções, quanto aos usuários, que não conseguem ter suas necessidades atendidas, dificultando o processo de cuidado.

Para o controle da pressão arterial, é fundamental a adesão ao tratamento farmacológico e não farmacológico. Mudanças de hábitos e estilo de vida podem ser drásticas e de difícil adaptação, com grandes impactos e consequências nas vidas dos portadores de hipertensão arterial (BARBOSA MEM, et al., 2019).

Almeida TCF, et al. (2019) relatou uma pequena proporção de indivíduos com níveis pressóricos adequados no grupo estudado, ainda que a maioria estivesse ciente das vantagens de aderir ao tratamento prescrito. A falta de adesão, bem como a adesão efetiva, pode estar associada às crenças positivas e negativas criadas pelo indivíduo hipertenso. Portanto, é indispensável ao profissional reconhecer crenças e comportamentos que devem ser descontruídos ou potencializados para favorecer tratamento e controle da hipertensão.

A percepção dos participantes em relação ao atendimento da equipe levantada na pesquisa de Gouveia Neto JR, et al. (2019) revelou que os usuários hipertensos que se disseram insatisfeitos com o atendimento, utilizaram como justificativa a falta de medicações e a dificuldade na marcação de exames, o que converge com os pontos apresentados pelos profissionais.

Quanto à resolução do questionário, houve um maior número de erros nas questões referentes à prevalência e classificação da hipertensão, ao procedimento de primeira verificação da PA e aos critérios para o fechamento do diagnóstico. Estes dados são incisivos, pois falhas nestes aspectos podem levar a diagnósticos incorretos. A mais nova classificação apresentada pela VII Diretriz Brasileira de Hipertensão utiliza os seguintes critérios: medidas menores ou iguais a $120 \times 80 \mathrm{mmHg}$ são consideradas normais, maiores que $120 \times 80 \mathrm{mmHg}$ caracterizam uma pré hipertensão, medidas que variam de $140-159 \times 90-99 \mathrm{mmHg}$ hipertensão grau I, 160-179x100-1009 hipertensão grau II, e hipertensão grau III medidas maiores que 180x100mmHg (MALACHIAS MVB, et al., 2016).

Para o fechamento do diagnóstico recomenda-se um mínimo de três aferições em dias distintos com equipamentos e preparo do paciente adequado. A primeira medida da PA deve ser realizada em ambos os braços e as subsequentes naquele que se obteve o maior valor (BRASIL, 2013).

Estudo de Melo LM, et al. (2015), afirma que as ações do cuidado necessitam de embasamento teóricocientífico, como o proposto por protocolos e diretrizes clínicas, uma vez que a efetividade e eficiência da assistência dependem da equipe multiprofissional e de fatores organizacionais dos serviços de saúde. Assim, os profissionais devem manter-se atualizados considerando as constantes mudanças no meio científico e o processo de trabalho avaliado e refletido, de maneira a adequá-lo da melhor maneira possível à realidade em que usuários e profissionais estão inseridos. E, nesse contexto, a Educação Permanente em Saúde, proposta de prática pedagógica que coloca o cotidiano do trabalho sob problematização, viabiliza a reconstrução de atitudes, modos de produzir e de se relacionar.

A pesquisa expôs uma experiência de atualização para equipe de saúde em diabetes mellitus por meio de oficinas educativas que constatou melhorias na qualificação dos profissionais, no intuito de uniformizar e sistematizar o atendimento ao usuário com diabetes em termos de integralidade, educação em saúde e desenvolvimento do autogerenciamento (DOSSE C, 2009). Os benefícios da educação permanente corroboram para o alcance da integralidade, promovem a formação crítica e reflexiva dos profissionais, e aproximam a gestão das questões locais de saúde, comprovando que pode ser considerada uma prática viável (YAMAMOTO TS, et al., 2015).

A escolha por metodologias ativas rompe práticas educativas tradicionais, e de forma leve consegue suscitar interesse. Baseadas na ação-reflexão-ação propicia a participação ativa dos envolvidos, a problematização do cotidiano e associação entre teoria e prática (PINHEIRO GEW, et al., 2018). As expectativas em utilizar este tipo de metodologias foram alcançadas, pois proporcionaram discussão, interação e reflexão da prática. Observa-se que a educação é inerente à organização do trabalho em saúde frente às mudanças técnico-científicas, as novas tecnologias, as mudanças sociais e epidemiológicas e ainda devido à complexidade e subjetividade do processo saúde doença e das relações entre profissionais e usuários (SILVA JAMD e PEDUZZI M, 2011). 
Em vista disso, o investimento em educação profissional é uma excelente estratégia, capaz de proporcionar ações com potencial de transformação e preenchimento de lacunas no cenário da saúde, a saber, a utilização de rodas de conversa com o apoio de cartilha ilustrativa.

Ao comparar o número de acertos antes e após as ações de intervenção, evidenciou-se que as metodologias utilizadas como formas de educação permanente foram capazes de aumentar o aprendizado por promover melhora do nível de conhecimento dos profissionais, destacando a viabilidade de ações deste tipo para qualificação profissional.

Como limitação, este estudo não conseguiu atingir a mesma população nos dois momentos por recusa ou indisponibilidade, visto que a coleta de dados ocorreu em horário de trabalho, embora com várias tentativas, não sendo possível avaliar o conhecimento e as ações de maneira uniforme.

Escassez de evidências quem comprovem experiências que abordam educação permanente em hipertensão com os profissionais que acompanham os usuários com hipertensão arterial, pode ser considerada outra limitação para a discussão dos dados. Sugere-se, portanto, o desenvolvimento de novas pesquisas na área destinadas aos profissionais.

Considerando a competência da APS em prevenir, controlar e monitorar a hipertensão, faz-se necessário enfatizar a importância de profissionais devidamente habilitados e aptos para lidar com essa demanda. Assim, espera-se que os resultados desta pesquisa contribuam para a elaboração de medidas que possibilitem a qualificação dos profissionais, sobretudo da APS, visto que o conhecimento garante a melhoria à assistência, e dessa maneira, se torna possível atingir adesão terapêutica efetiva, redução de complicações e de mortalidade por hipertensão.

\section{CONCLUSÃO}

Observou-se aumento no nível de conhecimento para todos os aspectos abordados na roda de conversa com aplicação de cartilha educativa: definição, dados epidemiológicos, diagnóstico e classificação, tratamento e acompanhamento da hipertensão. Tal achado evidencia o efeito positivo da estratégia utilizada, ao passo que destaca a necessidade de educação permanente de profissionais de saúde para qualificação do atendimento ao paciente hipertenso.

\section{REFERÊNCIAS}

1. ALMEIDA TCF, et al. Crenças de indivíduos com hipertensão arterial sistêmica relacionadas ao tratamento medicamentoso. Rev. Rene (online), 2019; 20: e41585-e41585.

2. BARBOSA MEM, et al. Fatores associados à adesão de adultos/idosos ao tratamento da hipertensão arterial na atenção básica. Rev enferm UERJ, Rio de Janeiro, 2019; 27.

3. BRASIL. Ministério da Saúde. Secretaria de Atenção à Saúde. Estratégias para o cuidado da pessoa com doença crônica. $1^{\circ}$ ed. Ministério da Saúde, Brasília, Brasil. 2014; 162 p.

4. BRASIL. Ministério da Saúde. Secretaria de Atenção à Saúde. Estratégias para o cuidado da pessoa com doença crônica: hipertensão arterial sistêmica. ${ }^{\circ}$ ed. Ministério da Saúde, Brasília, Brasil. 2013; 130 p.

5. BRASIL. Ministério da Saúde. Portaria no 198 GM/MS, de 13 de fevereiro de 2004. Institui a Política Nacional de Educação Permanente em Saúde como estratégia do Sistema Único de Saúde para a formação e o desenvolvimento de trabalhadores para o setor e dá outras providências. Diário Oficial da União. 2004.

6. CAMARGO RAAD, et al. Estratégia saúde da família nas ações primárias de saúde ao portador de hipertensão arterial sistêmica. Rev Min Enferm, 2013; 17(4): 864-881.

7. CODECOM. Programa Saúde em Casa entrega medicamentos a quase 10 mil pessoas em Campina. Campina Grande: Prefeitura Municipal de Campina Grande, 2017.

8. DOSSE C, et al. Fatores associados a não adesão dos pacientes ao tratamento de hipertensão arterial. Rev. LatinoAm. Enfermagem, 2009; 17(2): 201-206.

9. FALKENBERG MB, et al. Educação em saúde e educação na saúde: conceitos e implicações para a saúde coletiva. Revista Ciência \& Saúde Coletiva, 2014; 19(3): 847-852.

10. GOUVEIA NETO JR, et al. Adesão terapêutica e qualidade de vida de hipertensos assistidos na atenção primária de saúde. Nursing, Säo Paulo, 2019; 22(249): 2600-2603.

11. MALACHIAS MVB, et al. $7^{\mathrm{a}}$ Diretriz brasileira de hipertensão arterial. Arq Bras Cardiol, 2016; 107(3): 1-103.

12. MELO JD, et al. Hipertensão arterial sistêmica e fatores associados na Estratégia Saúde da Família em Imbituba/SC. Revista da AMRIGS, 2016; 60(2): 108-114. 
13. MELO LM, et al. Atuação do enfermeiro a pessoa hipertensa na estratégia de saúde da família: revisão integrativa. CuidArte Enfermagem, 2015; 9(2): 160-170.

14. MENDES OLM, et al. Fatores associados a não adesão ao tratamento da hipertensão arterial sistêmica: uma revisão integrativa. Revista Univap, 2014; 20(35): 56-68.

15. PESSOA MS, et al. Characterization of hypertensens at a basic family health unit. Revista International Journal of Development Research, 2018; 8(3): 19649-19653.

16. PINHEIRO GEW, et al. Facilidades e dificuldades vivenciadas na Educação Permanente em Saúde, na Estratégia Saúde da Família. Saúde em debate, 2018; 42: 187-197.

17. RODRIGUES ACS, et al. A proposta da educação permanente em saúde na atualização da equipe de saúde em diabetes mellitus. Rev. Esc. Enferm. USP, 2010; 44(2): 531-37.

18. SILVA JAMD, PEDUZZI M. Educação no trabalho na atenção primária à saúde: interfaces entre a educação permanente em saúde e o agir comunicativo. Revista Saúde e Sociedade, 2011; 20(4): 1018-1032.

19. TRIPP D. Pesquisa-ação: uma introdução metodológica. Educação e pesquisa, 2005; 31(3): 443-466.

20. YAMAMOTO TS, et al. Educação permanente em saúde como prática avaliativa amistosa à integralidade em Teresópolis, Rio de Janeiro. Revista Trab. educ. saúde, 2015; 13(3): 617-638. 